\title{
Improving proactive decision making with object trend displays
}

\author{
Alex Eftyhiou \\ Department of Computer Science \\ University College London \\ NW1 2FD, UK \\ a.eftyhiou@ucl.ac.uk
}

\author{
John Dowell \\ Department of Computer Science \\ University College London \\ NW1 2FD, UK \\ j.dowell@ucl.ac.uk
}

\begin{abstract}
Operators of dynamic systems often use time-series data to support their diagnostic and proactive decision-making. Those data have traditionally been displayed in the form of separate trend charts, for example, line graphs of pressure and temperature over time. Configural object displays are a widely advocated approach to the visual integration of information yet have been applied only rarely to time-series data. One example was the 'time tunnel' format but its benefits were equivocal, seemingly compromised by its graphical complexity. There is then the need to investigate other graphical forms for object displays of time series data. This research will require a microworld representing a knowledge-rich task domain accessible to multiple participants (the nuclear power plant simulation used with the time tunnel display studies required participants to have 20 hours of experience with the system). We report a design for such a microworld that adopts the domain of financial control of a business where decisions need to be made about the pricing of products to optimize returns in a changing and sometimes volatile market. Alternative visual displays of the essential time series data for this domain are possible and whilst decision making is knowledge rich, involving reasoning about high level relationships, pilot tests showed that it is accessible to participants with only moderate training.

\section{CCS Concepts}

Human-centered computing - Human computer interaction (HCI) - HCI design and evaluation methods - Laboratory experiments.

Interaction paradigms - Graphical user interfaces.

Author Keywords

Microworld; Configural object Visualisation; line graphs;

Decision-making Situation Awareness; Mental Models;

Ecological Interface Design.
\end{abstract}

\section{BACKGROUND}

User interfaces typically display continuously varying data in the form of line graphs, for example, the separate charts of pressure, temperature and mass flow that a control room operator might see. This format has been found to help detect trends and make predictions, particularly when the data being presented is static Bennett, Payne \& Walters 2005); when the data is real time and being displayed dynamically, the format has been found to produce less optimal effects (Spenkelink, 1990). Moreover, tasks that require operators or users to integrate SAMPLE: Permission to make digital or hard copies of all or part of this work for personal or classroom use is granted without fee provided that copies are not made or distributed for profit or commercial advantage and that copies bear this notice and the full citation on the first page. To copy otherwise, or republish, to post on servers or to redistribute to lists, requires prior specific permission and/or a fee.

Conference'10, Month 1-2, 2010, City, State, Country.

Copyright 2010 ACM 1-58113-000-0/00/0010 _..\$15.00.

DOI: http://dx.doi.org/10.1145/12345.67890 multiple temporal data sources are not well supported by line graphs. An alternative is the configural display, a graphical format intended to support this kind of integration in tasks. Configural displays use composite objects to represent different data elements and have emergent properties that represent the combination of parameters; for example, a square whose sides represented two variables (e.g, aperture and shutter speed) would have an area representing the integration of these variables (e.g., luminance); area in this example would be an emergent property of the square. Other examples of emergent features of visual objects include symmetry and orientation (Pomerantz, 1986). Configural displays are designed based on a detailed analysis of the work domain with the aim of the configural display acting as a representative externalised mental model. In an investigation by Burns (2008), the authors found that configural displays improved high level situation awareness (SA), which is linked to making accurate predictions about system states, over traditional and advanced displays. Mogford (1997) explains that "SA is the transient contents of awareness, as structured and supported by an underlying mental model". Therefore these externalised mental models presented as configural object displays hold promise for domains that involve prediction and proactive decision making. In particular we are interested in the proactive control of dynamic systems where it is important to provide operators with a historical trend otherwise known as a temporal context. While reviewing the literature on the integration of temporal contexts with configural object displays, we only uncovered two different formats.

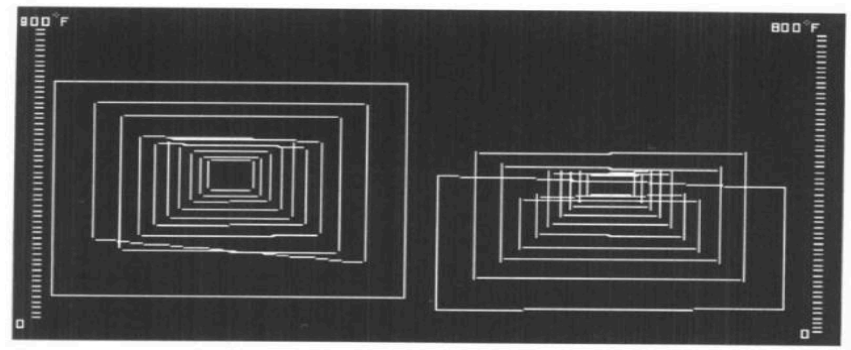

Figure 1: The time tunnel display (from Hansen, 1995)

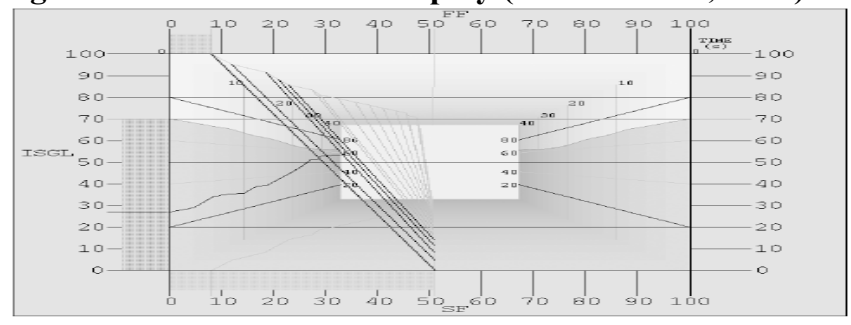

Figure 2: The time tunnel display Bennett, Payne \& Walters (2005) 
The first is known as the time tunnel developed by Hansen (1995), Bennett \& Zimmerman (2001), Bennett, Payne \& Walters (2005) where temporal data is integrated with the configural object in a continuous manner, the succession of frames receding in perspective view representing systems states at consecutive intervals can be seen in figure 1 and figure 2 . Three investigations were conducted on the time tunnel format, the first by Hansen (1995) who looked at differences between configural object displays and separable displays for a simulated process monitoring task to detect when eight variables associated with the corners of the two boxes were in a state of decline. The study required relatively short training because the task involved only perceptual judgements of the graphical displays and not decisions requiring domain knowledge. By contrast, Bennett \& Zimmerman (2001) used the time tunnel format to investigate diagnostic and predictive decision-making that did require domain knowledge. Participants had a minimum of 20 hours experience in similar experiments and were given a further hour of training. Participants were required to carry out fault detection tasks and were asked about high-level properties such as the relationships between the variables along with low-level properties involving individual variables. Bennett, Payne \& Walters (2005) also used the same time tunnel format to investigate fault detection and prediction. Participants were selected who had a minimum of 30 hours experience with the microworld and received a one hour refresher training course.

There were issues with how perceptible and thus useful the historical context was to participants with this approach. In the Hansen (1995) study participants attended to the most recent system state, stored it in working memory and built their own mental trend that was more readily available. Bennett and Zimmerman (2001) and Bennett, Payne \& Walters (2005) also took the time tunnel approach as demonstrated in figure 2 and found that participants adopted similar strategies. As temporal contexts are part of operator mental models when controlling dynamic systems and operators are adopting a configural object display as their externalised mental model, then the display should also ensure that temporal contexts are perceivable and effectively integrated into the configural graphic. Otherwise the display may be problematic in that it struggles to act as an externalised mental model since it is not representing the crucial time element properly. Based on Mogford (1997) these aforementioned externalised mental model issues could undermine SA and thus projections of future states that are advantageous to proactive decision making. It is therefore important that we understand how integration of the temporal element with configural object displays can be advantageous, but also what to look out for.

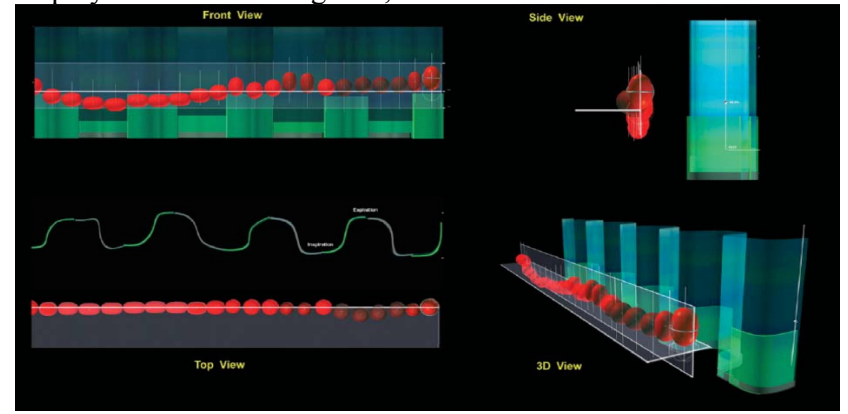

Figure 3: Discrete object trend (Zhang et al, 2002)

The second example of an attempt to integrate temporal context with a configural object (figure 3) was developed by Zhang et al (2002) for the anesthesiology domain where system states are presented as discrete points in time. Similar to the Hansen (1995) study, participants were asked to make perceptual judgements about the display and not clinical decisions about the patient that the displayed data represented. This protocol was inevitable given the unavailability of specialist anesthetists and the use of medical students instead as participants. Bennett \& Zimmerman (2001), Bennett, Payne \& Walters (2005) and Zhang et al (2002) studies created significant barriers for participant selection because of the highly specialist domains selected for their microworlds. They illustrate the need for a microworld for investigating configural object displays with temporal contexts where non-specialist participants are able, with moderate training, to use domain knowledge to make diagnostic and predictive decisions. A more accessible micro-world would assist data collection of various configural graphics with temporal contexts and inform display design in order to effectively integrate the temporal context. Data collected can also support design by aiding our reasoning about relevant cognitive constructs such as mental models of complex dynamic systems.

\section{THE MICROWORLD DOMAIN}

We have developed a microworld for investigating proactive decision making with object trend displays. It presents a domain that is accessible to participants with moderate training but which nevertheless requires knowledge-rich decision making. The domain is the financial management of a commercial enterprise operating in changeable market conditions and with changeable production conditions that determine how the input variable of price relates to the outcome variable of profit. The company sets the price of its product in successive daily intervals so that prediction of future market conditions is key to optimizing profits. Participants are given 12 days' worth of data on price, sales, costs, revenue and profit patterns in order to diagnose the underlying conditions in the environment and decide on the future price points for the product. All variables in the micro-world are coupled either directly or indirectly through other variables and have either inverse or direct relationships. Price is coupled to profits through the variables of sales and cost. The variable of sales reflects number of items produced as products are made to order. Sales have a dynamic inverse relationship with price. The variable cost per item made has a dynamic inverse relationship with sales and therefore cost also has a direct dynamic relationship with price. The dynamic micro-economy domain focuses on the relationships between variables. Object displays with emergent features permit users to directly perceive higher order emergent properties of the domain and reason effectively about the relationships between variables. The knowledge rich environment is hierarchical in order to examine the different levels that are expressed in configural graphics that range from low level variable detection change to integration, reasoning and diagnosis about the meaning of higher order relationships between the multiple connected variables. Participants are required to make diagnostic judgements about the relationships and identify relationship patterns to support proactive decisions about how to manipulate the input variable in order to optimize an outcome variable. To do this, participants must recognize the changing relationships between both directly and indirectly related input variables and output variables.

\subsection{Dynamic relationship between price and sales.}

The sales variable will change in how it interacts with the input variable of price. We have already stated the inverse relationship between price and sales. Further this inverse relationship is dynamic being either elastic or inelastic which is an effect companies experience within the real market depending on factors 
such as number of substitute products in the market and customer spending power. To aid participants the terms elastic and inelastic were replaced with responsive and unresponsive. So for example if the relationship is between price and sales is responsive then a change in price will cause a relatively larger change in sales, further the way we have set up the system means that in a responsive condition a decrease in price will increase the emergent variable of revenue, which is beneficial to the profit optimisation. If the relationship is unresponsive then a change in price causes a relatively smaller change in sales. In the unresponsive condition an increase in price will cause a beneficial increase in revenue. How responsive sales are to this change in price can change each day depending on the market, thus there is a dynamic relationship between price and the sales variable which in turn affects the revenue created by a particular price setting.

\subsection{Dynamic relationship between sales and cost.}

The cost to produce a single product typically has an inverse relationship with sales in that higher sales will lower the cost per item produced and vice versa. We have based this on an economy of scale effect where higher production creates increased economies of scale bringing down total costs since in our company sales reflect the number of products made. The effects of economies of scale are dynamic both increasing in their effect and diminishing over time meaning that different days will have a different cost to sale responsiveness. The inverse relationship between price and sales and the inverse relationship between sales and cost leads to the fact that price and cost are directly related so a change in price creates the same change in cost. In cases where cost per item is highly responsive to sales, an increase in price per item will cause a greater increase in cost per item. This in turn will decrease profits even though that particular price change might be increasing revenue. Cost per item is multiplied by the sales to give the emergent property of total costs.

\subsection{Combining Revenue and Total Cost to give the profit emergent feature.}

Revenue and Total costs are subtracted in order to give the profit emergent property. As all variables are coupled the cost per item variable is also relevant to price changes as discussed in the previous section. Therefore participants cannot simply attend to increasing revenue so as to increase profit. They need to be aware of how price per product affects the total costs based on the cost per product and sales relationship.

\section{DISCRETE TEMPORAL PRESENTATION OF THE CONFIGURAL OBJECT}

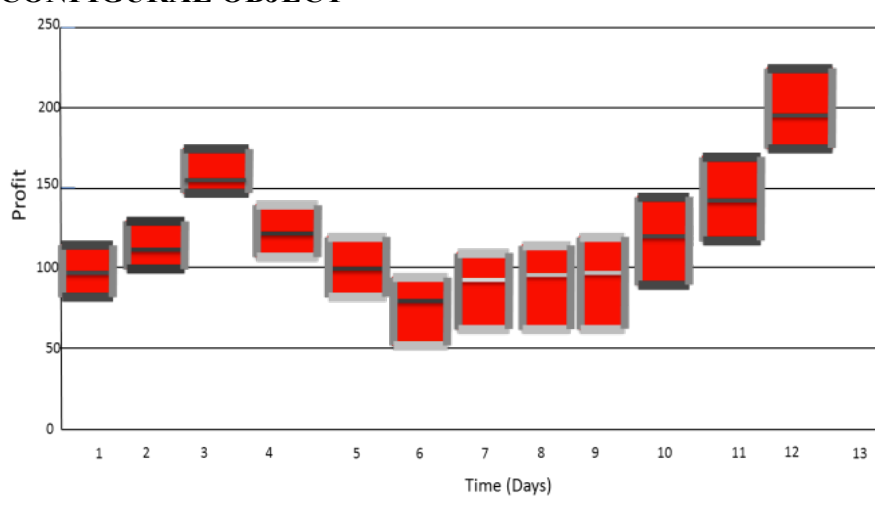

Figure 4: Discrete object trend graphic.

A primary requirement for this micro-world was that it should be accessible to non-specialists who, with moderate training, would be able to make diagnostic and predictive decisions. Our pilot studies have demonstrated that this requirement is achieved. Our micro-world has currently adopted the discrete object visualization used in Zhang et al (2002). However continuous displays similar to the time tunnel can also be investigated with this microworld. The microworld is supporting investigations into object visualisations of time series and trend data that can achieve the same advantages and support participants' reasoning about higher-level relationships and decisions with temporal configural displays. The above graphic is comprised of heterogeneous codes of colour and form (Wickens and Carswell 1995). Variable discrimination and perception of variable relationships are enhanced by colour coding, while emergent features are identifiable by configuration of lines. We have followed these proximity compatibility guidelines while coherently semantically mapping the graphic to the microworld domain. We are going to represent the micro-world using a discrete form of object visualization (following Zhang et al, 2002) where the temporal context is presented discretely. Zhang (2002) had to devolve the participant task to surface level detection as opposed to reasoning about variable relationship meanings to diagnose and treat patients. We will therefore take the opportunity to now test this format with a more sophisticated task. In the above visualization the price determines the height of the square configural object while sales determine the width of the object. The horizontal line running through the object is the cost line, note that the area below the cost line represents emergent property of total costs incurred while the area above represents the emergent property of total profit made. The object is positioned on the graph according to total profit. The emergent property of revenue (area inside box) is colour coded to enhance participants in determining increases (coloured red) or decreases (coloured blue) in revenue.

\subsection{Price and Sales visual coding}

Note that different shades of grey are located on the object perimeter. The shading on the vertical sides of the box represent price, as price is the control variable and is not affected by other variables, the price shading remains unchanged. However the sales shading changes depending on responsiveness. In cases where sales are responsive to price changes as is the case in day 13 and $10-12$, the horizontal sides of the boxes are shaded darker than price. However in cases where sales are unresponsive to changes in price as is the case from day 4-9 the horizontal sides are a lighter shade than price. Therefore participants are supported in their reasoning about the relationship status on a given day.

\subsection{Sales and Cost visual coding}

Participants are required to compare the fill shading of the cost line running through the box object with the horizontal sides representing sales. In cases where costs are responsive to sales the cost line will be shaded darker than the sales line while the cost line is lighter for when unresponsive to sales. If costs are responsive to sales then a fall in sales will cause an increase in total costs, whereas in cases where costs are unresponsive to sales an increase in sales will cause an increase in total costs. This is particularly problematic if the responsiveness of the cost line to the sales is such that the increase in cost is such that it outpaces an increase in the price or a decrease in cost is slower than a decrease in price. On days 3-5 on figure 4 we can see that price increase as shown by the increase in object height is causing a significant increase vertical positioning of the cost line. The cost line increasing faster than the price increase over the next three day period. The effect that this has is that the price increase required to optimize revenue in this unresponsive sales to price period is now having an adverse effect on the profit as demonstrated by the decrease in vertical positioning (profit) of the object over day 3-5. 
This greater change in cost than price is represented by the fact that the cost line is shaded darker than the price in days 3-5. Participants therefore need to consider all variable relationships both direct and indirect before setting the price change they believe will optimise profits.

\subsection{Separated display}

The comparison display format participants use is separable charts, comprising of 7 individual line charts. This display is a baseline from which to judge the effect of the discrete object display on participants' ability to integrate the several variables as they need to do to perform the diagnostic and predictive tasks they are given.

\section{PARTICIPANT TREND DIAGNOSIS, PREDICTION AND ACTION.}

To facilitate task completion participants have to order preset descriptors of ongoing variable relationship types for different sections of the trend. If participants are able to arrange the preset options in the same order as the trend they are informed that the trend expresses three repeating variable relationship conditions that each last for three day periods and will be asked which one they believe will repeat on day 13-15. For example in figure 4 the conditions of day 4-6 will repeat on day 13-15, although this does not mean that objects on day 13-15 will be identical to days 4-6 as variable values will have changed. What will repeat are the conditions that bring about the same variable relationships the company experienced on day 4-6. On day 4-6 an increase in price caused a decrease in profits participants must therefore decrease price on day 13-15 to optimize profit. Pilot participants were able to grasp the concept of how variable relationships determined how price should be changed to optimize profits. They were also able to correctly order the preset options after moderate training of 3 minutes in the object display visualization condition, however the separable condition created problems due to the necessity of integrating seven displays with multiple relationships between them.

To facilitate task completion participants must choose from preset options that describe different parts of the trend. These can be as follows:

Trend Part 1: Responsive market conditions due to numerous competitors entering the market and responsive economies of scale.

Trend part 2: Unresponsive conditions due to diminishing competition but highly responsive economies of scale effects.

Trend part 3: Unresponsive conditions continue with sales levels causing economies of scale effects to diminish.

Trend Part 4: Responsive market conditions due to numerous competitors entering the market and economies of scale effects increased.

The order of the above pre-set options are in line with the trend sections in figure 4, however in a task situation the preset options would be randomized and participants would be asked to put them in the correct order as described by the trend. This allows us to determine whether participants in fact understand the relationship types between variables described by the trend.

With the microworld we will collect data relating to decision accuracy, reaction times, qualitative probes, task load, confidence ratings. These measures will enable us to examine cognitive constructs such as mental models along with the human performance, for different forms of configural displays with temporal contexts. We are also interested in examining biases that may be induced by the different manipulations of the configural object displays and temporal contexts. This is an important consideration since, if the operator comes to rely on the display as their mental model, it may be more challenging for them to detect discrepancies as the only mental model they hold is the one they are looking at on the screen. Also we are interested in taking measures of SA and in particular high level SA relating to the projection of future states as this is relevant to proactive decision making. We are currently implementing the discrete display, following Zhang et al (2002), but continuous displays similar to the time tunnel can also be investigated with this microworld.

We intend to increase the knowledge base regarding these types of displays so as to assist with improved integration of the temporal context with configural object displays and to also understand how different design manipulations may be more appropriate than others given different types of task types.

\section{CONCLUSION}

A primary requirement for this microworld was that it should be accessible to non-specialists who, with moderate training, would be able to make diagnostic and predictive decisions. Pilot studies have demonstrated that this requirement is achieved. The microworld is supporting investigations into object visualisations of time series and trend data that can achieve the same advantages already found for object visualisations of aggregate and static data.

\section{REFERENCES}

[1] Bennett, K. B., Payne, M., \& Walters, B. 2005. An evaluation of a "time tunnel" display format for the presentation of temporal information. Human Factors, 47, $342-359$.

[2] Hansen, J. P. 1995. An experimental investigation of configural, digital, and temporal information on process displays. Human Factors, 37, 539-552.

[3] C. M. Burns, G. Skraaning, Jr., G. A. Jamieson, N. Lau, J. Kwok, R. Welch, and G. Andresen. 2008 "Evaluation of ecological interface design for nuclear process control: Situation awareness effects," Human Factors, vol. 50, pp. 663-679,

[4] Mogford, R. H. 1997. Mental models and situation awareness in air traffic control. International Journal of Aviation Psychology, 7, 331-342.

[5] Pomerantz, J. R. 1986. Visual form perception: An overview. In H. C. Nusbaum \& E. C. Schwab (Eds.), Pattern recognition by humans and machines: Volume 2 . Visual perception (pp. 1-30). Orlando, FL: Academic Press.

[6] Spenkelink, G. P. J. 1990. Aiding the operator's anticipatory behaviour: The design of process state information. Applied Ergonomics, 21, 199-206.

[7] Wickens, C.D., Carswell, C.M. 1995. The proximity compatibility principle: Its psychological foundation and relevance to display design. Human Factors, 37, 473-494.

[8] Zhang Y, Drews F, Westenskow D, Foresti S, Agutter J, Bermudez J, Blike G, Loeb R. 2002 Effects of integrated graphical displays on situation awareness in anaesthesiology. Cogn Technol Work 4, 82-9. 